\title{
EXPERIENCIAS EN LA CO-CONSTRUCCIÓN DEL PATRIMONIO EN EL ALTO
} (CATAMARCA, ARGENTINA)

HERITAGE'S CO-CONSTRUCTION EXPERIENCES IN EL ALTO (CATAMARCA, ARGENTINA)

Inés Gordillo

Luciana Eguia

Verónica Zuccarelli

Carolina Prieto

Sebastían Bocelli

José Miguel Letelier

Hector Buono

Como citar este artigo:

GORDILLO, Inés et al. Experiencias en la co-construcción del patrimonio en El Alto (Catamarca, Argentina). Cadernos do Lepaarq, v. XVII, n.34, p. 146-163, Jul-Dez. 2020. 


\title{
Experiencias en la co-construcción del patrimonio en El Alto (Catamarca, Argentina)
}

\author{
Inés Gordillo ${ }^{\mathrm{a}}$ \\ Luciana Eguia $^{\mathrm{b}}$ \\ Verónica Zuccarelli ${ }^{\mathrm{c}}$ \\ Carolina Prieto ${ }^{\mathrm{d}}$ \\ Sebastían Bocelli ${ }^{\mathrm{e}}$ \\ José Miguel Letelier ${ }^{\mathrm{f}}$ \\ Hector Buono ${ }^{\mathrm{g}}$
}

\section{Resumen:}

En este trabajo presentamos el avance de dos proyectos de aplicación vinculados a la visibilización, valoración y activación patrimonial, desarrollados en las localidades de Guayamba y Tapso, Dpto. de El Alto, Catamarca, Argentina. Para lograr nuestros objetivos planteamos tres ejes articuladores: una noción ampliada de patrimonio basada en el concepto de paisaje cultural, una construcción en conjunto con las comunidades locales y la integración de estos ejes con el turismo autogestionado y sustentable.

\section{Palabras-clave:}

Patrimonio, Paisaje, Catamarca.

\begin{abstract}
:
In this paper we address two projects in progress developed in the towns of Guayamba and Tapso, Department of El Alto, Catamarca, Argentina. The aim was to explore the visibility, valuation and activation of heritage policies. To achieve our goals, we propose three articulated items: an expanded notion of heritage based on the concept of cultural landscape, a co-construction in collaboration with local communities and the integration of these items with self-managed and sustainable tourism.
\end{abstract}

\section{Keywords:}

Heritage, Landscape, Catamarca.

\footnotetext{
${ }^{a}$ Dra. Instituto de Arqueología, FFyL, Universidad de Buenos Aires.

${ }^{\mathrm{b}}$ Dra. CONICET- Instituto de Arqueología, FFyL, Universidad de Buenos Aires.

${ }^{\mathrm{c}}$ Dra. CONICET- CITCA/Universidad Nacional de Catamarca.

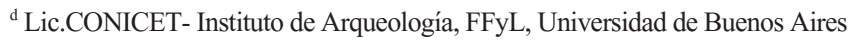

${ }^{\text {e }}$ Prof. CONICET- CITCA/Universidad Nacional de Catamarca.

${ }^{\mathrm{f}}$ Instituto de Arqueología, FFyL, Universidad de Buenos Aires

${ }^{\mathrm{g}}$ Instituto de Arqueología, FFyL, Universidad de Buenos Aires
} 


\section{INTRODUCCIÓN}

En este artículo nos proponemos narrar nuestras experiencias de trabajo sobre el patrimonio en las localidades de Tapso y Guayamba, ubicadas en el departamento El Alto, provincia de Catamarca, Argentina (figura 1). Desde hace una década venimos realizando investigaciones arqueológicas en la zona, pero fue en el transcurso de los últimos años cuando empezamos a dar forma a dos proyectos de aplicación vinculados a la visibilización, valoración y activación patrimonial. Ambas iniciativas tuvieron como eje central el trabajo conjunto y mancomunado con las comunidades locales y sus diversos actores sociales (Gordillo 2017).

El oriente de Catamarca es un área crítica para comprender los procesos sociales ocurridos durante más de dos milenios en el Noroeste Argentino. Esto, sumado a las características particulares de la región, sus atractivos naturales y a la predisposición de su gente, han favorecido el diseño de un plan de manejo patrimonial integral, donde se promueva un producto turístico-cultural. Nuestra propuesta contempla circuitos en el terreno, centros de interpretación y programas de promoción y participación comunitaria. Nos interesa abrir las puertas a nuevas narrativas sobre la historia y el paisaje local, y comenzar a desandar la idea de que los únicos elementos patrimonializables son aquellos considerados de mayor jerarquía o envergadura. En este sentido, se busca poder reconocer aquellos elementos que resultan valiosos para las comunidades de la zona, en función de sus necesidades actuales. Al respecto, nos enriquecen las experiencias de esta índole que, durante las últimas décadas, se vienen realizando en nuestro país, las cuales rescatan las narrativas multivocales sobre el pasado a partir de la consideración de diferentes racionalidades (Jofré et al. 2008; Aschero et al. 2005; Korstanje et al. 2013; Delfino et al. 2013).

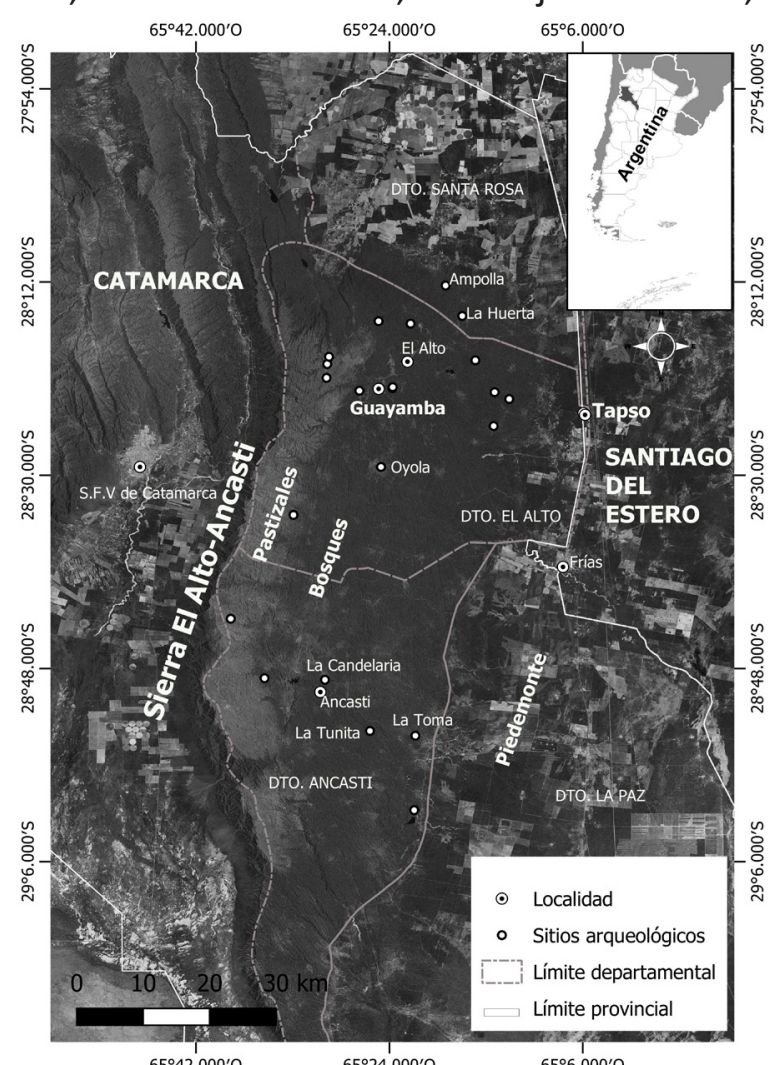

Figura 1: Mapa del área oriental de la provincia de Catamarca, Argentina. (Mapa base: Esri, Maxar, GeoEye, Earthstar Geographics, CNES/Airbus DS, USDA, USGS, AeroGRID, IGN, and the GIS User Community) 


\section{PATRIMONIOS Y ALGO MÁS}

Como muchas definiciones en el campo de las ciencias sociales, el patrimonio se encuentra en el centro de varios debates. Íntimamente ligado a otras definiciones, como la de cultura, y abordado por diversas disciplinas, organismos y actores, definirlo puede resultar complejo. Sin embargo, probablemente, allí resida su gran potencial.

Durante los últimos años estas discusiones llevaron a una lectura del patrimonio, y de su vínculo con la arqueología, que ya no puede ni quiere ser inocente (Argüello García 2019). El patrimonio nos convoca por su carácter construido, dialécticoy dinámico (Hernández-Ramírez 2007). Es contingentemente histórico, y también una construcción política; involucra diversos actores, que conviven e interactúan en función de diferentes fuerzas y focos de interés (Gnecco 2019; Londoño 2014).

Creemos que el patrimonio no es algo "dado", algo que realmente existió, no es parte de una historia objetiva o un objeto valioso per se, sino que refiere a los usos del pasado en el presente, respondiendo a formas vigentes que tienen las personas y los grupos de valorar objetos, prácticas y lugares (Alonso González 2016; Arantes 2007; Troncoso 2010). Son las comunidades, entidades dinámicas y multivocales, quienes definen y valoran el patrimonio a partir de la negociación y elaboración de sentido propio (Unesco 2003; Jofré et al. 2008; Mc David 2014).

Consideramos que la arqueología no "descubre" un pasado que existió, sino que lo interpreta. Asimismo, el discurso arqueológico es solo una de las narrativas posibles sobre el pasado, ni la mejor ni la más importante (Bellelli 2012). En este sentido, las comunidades que han transitado y habitado estos paisajes a través del tiempo, también poseen sus propias narrativas, y sus modos de vincular el pasado y el presente (Ingold 2000; Vaquer y Cámera 2018).

Por las dimensiones de las localidades aquí trabajadas resulta significativa la noción de patrimonio local (Prats 2004) en referencia a comunidades personalmente interrelacionadas, un mundo conocido de conocidos. En ellas los objetos, lugares y manifestaciones, patrimoniales o no, se vinculan intensamente con la biografía de los individuos y con sus interacciones. El patrimonio local funciona como un foro de la memoria, donde se manifiestan procesos de reflexión y proyección de la comunidad sobre soportes diversos que, partiendo de las preocupaciones y retos del presente, reflexiona sobre su pasado, para proyectar, participativamente, el futuro (Prats 2004).

El trabajo conjunto y paralelo con la comunidad, no es solo un requisito marcado por la UNESCO, es una decisión y una negociación constante. Incorporar las voces de diversos actores implica contemplar los intereses e ideas de todos. Por esta razón, proponemos una forma de trabajo donde la práctica arqueológica se centre en dos conceptos claves: la colaboración y la inclusión (Funari y Carvalho 2014). El objetivo consiste en ampliar el diálogo, trascendiendo los límites de la academia, generando una disciplina más inclusiva, donde haya una integración y una co-construcción del conocimiento con un público amplio, comprendido por diversos actores. Sin dejar de lado la diversidad interna, muchas veces confrontada (González Ruibal et al. 2018). Asimismo, se busca poder incorporar los diversos intereses, poniendo en valor y gestionando el patrimonio de forma conjunta con la comunidad, hacer arqueología con la gente (Criado Boado 2012). 
En este marco, el patrimonio no es solo un "recurso" factible de generar ingresos. Su activación es un modo de crear un compromiso social que promueve el bienestar comunal en función de sus necesidades, de modo tal que el rédito quede en mano de las comunidades (Bellelli 2012; Shackel 2014). Este proceso debe contemplar tanto las oportunidades y bienestar de la población, como los riesgos y amenazas que dicho proceso le puede acarrear.

Nuestras investigaciones se encuadran dentro de las Arqueologías del Paisaje, donde el paisaje adopta una perspectiva holística. Este se compone de un conjunto de relaciones entre sujetos y lugares, es una entidad relacional constituida por las personas en su compromiso con el mundo (Thomas 2001). Es habitado, pensado, imaginado y así se constituye como tal (Gordillo 2014). En estos términos, el espacio, y la experiencia en torno al mismo, no es neutral, sino que está permeada por diferentes tipos de relaciones de poder (Soler Segura 2007). Los paisajes se encuentran en permanente construcción y transformación, a partir de la interpretación de espacios previos (Barrett 1999; Ingold 2000). Todo paisaje contiene restos materiales pretéritos dentro de una red de interpretaciones coetáneas y sucesivas; su análisis nos permite abordar la forma en la que las sociedades se relacionan con su propio pasado (Gordillo y Vaquer 2013).

Este vínculo otorga un significado propio a cada lugar en función, no sólo de la ocupación del espacio, sino también de las actividades que son ejecutadas y su referencia a otros tiempos. Es así, que ciertos lugares pueden ser incorporados como "hitos del paisaje" de las sociedades siguientes. En este sentido, en los paisajes vigentes se incorporan determinadas expresiones y reinterpretaciones de los grupos precedentes.

Asimismo, en la gestión patrimonial el paisaje puede proponerse como unidad transversal de intervención, desplazando la atención desde elementos o sitios aislados hacia los paisajes culturales (Soler Segura 2007; Criado 2012; entre otros). Una unidad que no es el resultado directo de las acciones de los grupos humanos sobre su entorno natural ni una simple estratigráfica cultural, sino una integración de lugares, prácticas e interpretaciones que se estructuraron en distintos planos y lógicas espaciales dentro de un territorio (Gordillo 2014).

\section{ÁREA DE TRABAJO}

Nuestro trabajo se desarrolla en la región Este de la provincia de Catamarca, recorrida en dirección N-S por la sierra El Alto-Ancasti. Se corresponde con la vertiente oriental del área andina en el Noroeste argentino, un ámbito crucial en la dinámica social precolombina. La sierra comprende distintos ambientes: los Pastizales de Alturas (entre los 1400 y 1900 msnm), las Yungas o Bosque Serrano (700 y $1400 \mathrm{msnm}$ ) y los arbustales del Chaco Semiárido, hacia la llanura chaco-santiagueña. En relación a la demografía, esta no supera los 5.000 habitantes, concentrados mayormente en pequeños pueblos como El Alto, Guayamba, Tapso o Vilismán. La principal fuente laboral es el empleo público municipal y en menor medida, el comercio, la agricultura y la ganadería a pequeña escala. El turismo presenta una marcada estacionalidad y, en consecuencia, parte de la población y de la planta turística queda ociosa el resto del año. El desarrollo turístico se concentra principalmente en los balnearios de la localidad de 
Guayamba, siendo menos frecuente en otras áreas. En las últimas décadas el Este provincial muestra una tendencia en la disminución histórica de la tasa de crecimiento demográfica, sólo atenuada en algunas zonas del norte por su incorporación a la producción sojera. En general, la población joven emigra en busca de trabajo a centros urbanos de mayor envergadura, como las ciudades de Frías, Catamarca, Tucumán o Córdoba (Gordillo 2017).

Durante décadas, la sierra El Alto-Ancasti fue reconocida principalmente por las representaciones rupestres en cuevas, con iconografía asociada a sociedades del primer milenio de la era en el sector sur de la sierra, donde se destacan La Tunita, La Candelaria y La Toma (Llamazares 1997-1998; Nazar 2003; entre otros). Este foco en el arte rupestre se ha ampliado hacia otras prácticas diversas de la vida prehispánica. En este sentido, hemos llevado a cabo investigaciones regulares mediante prospecciones, relevamientos rupestres y excavaciones de sitios de habitación y espacios agrícolas en el sector norte de la sierra desde el año 2008 (Bocelli 2017; Gordillo et al. 2017; Eguia 2019; Zuccarelli 2020, etc).

En el sector de bosques, donde se ubica Guayamba (figura 2), pudimos distinguir 128 sitios de varias clases: arte rupestre (12), habitación (3), áreas de morteros (30) y estructuras de producción (70). Aquí realizamos excavaciones en áreas de vivienda vinculadas a cuevas con arte rupestre -fechadas entre los siglos VI y XI dC.- y prospecciones donde se localizaron estructuras agrícolas, morteros y conanas, lo que da cuenta de la complejidad de los paisajes prehispánicos. Los análisis de los materiales recuperados indican que allí se realizaron diversas prácticas propias de un sitio de habitación permanente: preparación y consumo de alimentos, elaboración de artefactos líticos, entre otras.

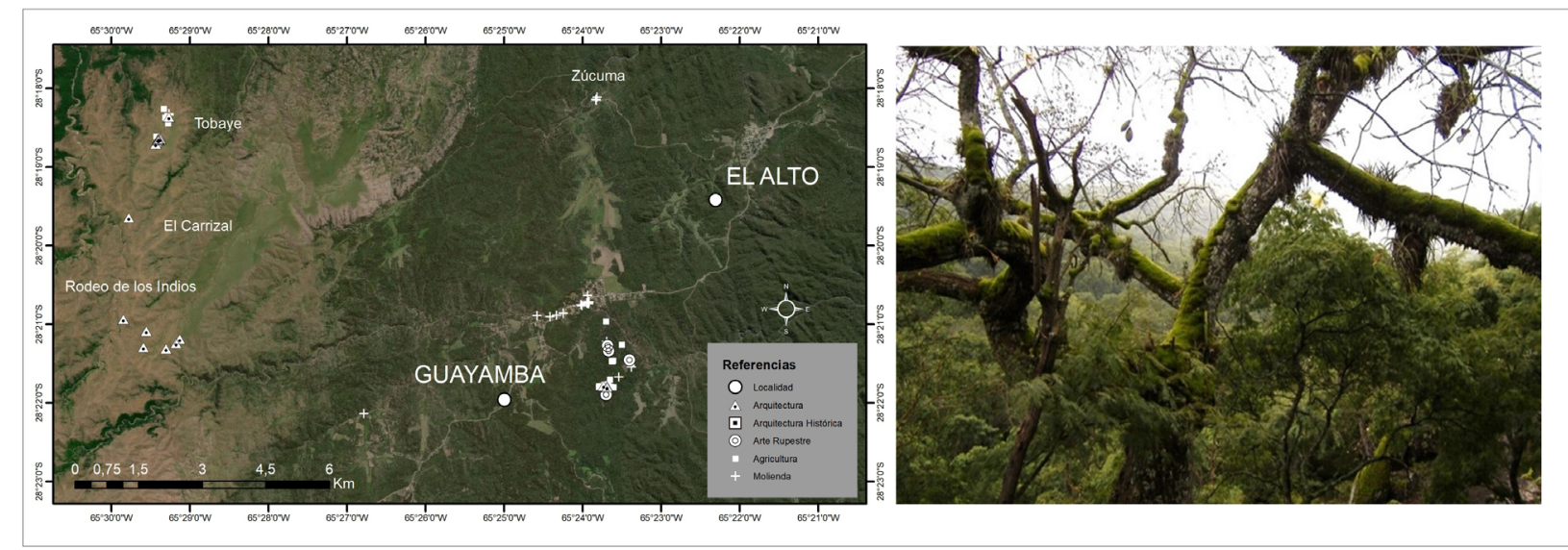

Figura 2: Mapa del área de Guayamba con distribución de sitios arqueológicos (izquierda) y foto del ambiente (derecha). (Mapa base: Esri, Maxar, GeoEye, Earthstar Geographics, CNES/Airbus DS, USDA, USGS, AeroGRID, IGN, and the GIS User Community)

En el Chaco-Semiárido, donde se encuentra la localidad de Tapso (figura 3), la evidencia arqueológica más conspicua identificada hasta el momento es el arte rupestre (18 sitios), seguida por áreas de molienda (14), arquitectura histórica (9) y prehispánica (2). Como resultado de nuestras prospecciones direccionadas por pobladores locales, registramos arte rupestre en tres sectores: EI Cajón, La Aguadita y Pozos Grandes (Gordillo et al. 2017; Bocelli 2017). Los estilos presentes, donde predominan diseños zoomorfos, muestran similitudes con los relevados en zonas aledañas como Los Algarrobales, La Huerta y Casa Pintada de Guayamba (Gordillo et al. 2017), Oyola (Gheco 2017) y las Sierras Centrales en el Norte de Córdoba (Recalde 2015). 

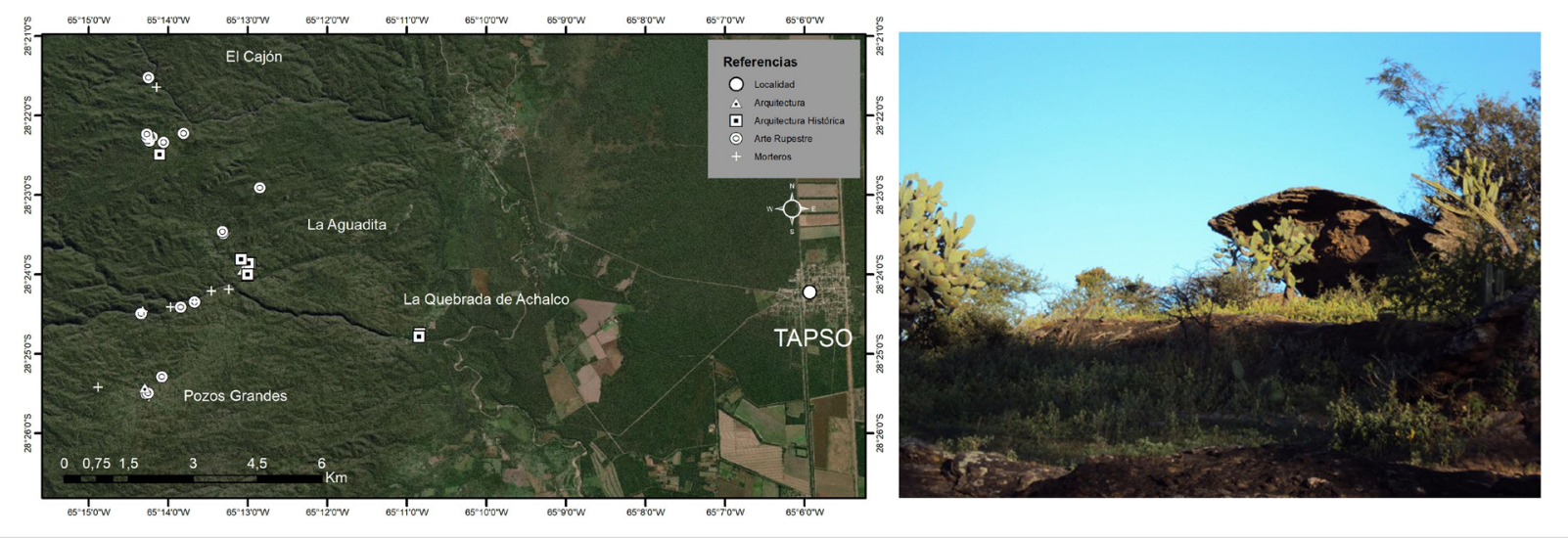

Figura 3: Mapa del área de Tapso con distribución de sitios arqueológicos (izquierda) y foto del ambiente (derecha) (Mapa base: Esri, Maxar, GeoEye, Earthstar Geographics, CNES/Airbus DS, USDA, USGS, AeroGRID, IGN, and the GIS User Community)

Tapso es una localidad que muestra aspectos identitarios que la distinguen de otras regiones; es un espacio de frontera con Santiago del Estero donde, cruzando una vía del tren, hay otra Tapso perteneciente a una provincia distinta. También es el lugar de nacimiento del gobernador de Catamarca Crisanto Gómez (Alaniz 2010), a la vez que posee figuras emblemáticas relacionadas con el turismo religioso que convergen con componentes indígenas prehispánicos. Con el impulso de la intendencia local, se han construido en los últimos años hitos arquitectónicos orientados a posicionarla como un lugar pintoresco y atractivo para turistas y peregrinos, como el Cristo redentor de 10 metros que se yergue en el centro geográfico del pueblo o la réplica de grandes dimensiones de "La Piedad" de Miguel Ángel próxima a la antigua iglesia de Achalco. Paralelamente, se inauguró el museo local y posteriormente se visibilizaron en el paisaje urbano elementos (carteles y murales) alusivos a la historia prehispánica.

\section{AVANCES Y SITUACIÓN ACTUAL DEL EMPRENDIMIENTO}

Nuestras actividades de aplicación social en la zona se enmarcaron en dos proyectos ${ }^{1}$ avalados por diferentes instituciones científicas de Argentina, con metas y líneas de acción comunes. Además de la investigación arqueológica de base, sus objetivos se orientan a la visibilización y valoración del patrimonio cultural, enfocando principalmente al arte rupestre de las dos áreas (Tapso y Guayamba) y dirigidos a la gestión del patrimonio cultural como fundamento para estimular la actividad turística sustentable. El desarrollo de ambos proyectos fue disímil. En Tapso, se pudieron concretar los avances más significativos, mientras que en el área de Guayamba estuvo más atado al pulso de las decisiones administrativas/financieras y políticas.

Paralelamente, en concordancia con la propuesta de reforzar el vínculo con las comunidades, decidimos desplazar actividades de intercambio académico, tradicionalmente radicadas en ciudades, a las localidades del área. Junto con el Equipo Interdisciplinario El Alto-Ancasti diseñamos el Taller de Arqueología de la Sierra de Ancasti y Zonas Aledañas (TASA), un espacio de discusión e intercambio

\footnotetext{
$1 \quad$ Se trata de los siguientes proyectos: 1) "El Arte Rupestre y la vida social a lo largo de dos milenios. Puesta en valor turístico del patrimonio cultural en el oriente de Catamarca". ASETUR, Ministerio de Ciencia, Tecnología e Innovación Productiva (MINCyT), Argentina, 2016; y 2) “Arte Rupestre y Patrimonio Cultural en Tapso (Depto. El Alto, Catamarca): Activación y Gestión del Turismo”. PDTS 143, Consejo Interuniversitario Nacional (CIN), Argentina, 2016.
} 
sobre avances de investigación regional de carácter periódico bianual, que tuvo su primera edición en Tapso. En el marco de estos talleres, se habilitaron espacios de encuentro con la comunidad y público en general, donde se compartieron los progresos y resultados de nuestros trabajos.

En el año 2019 ambos proyectos se vieron afectados por decisiones de índole burocrático que limitaron drásticamente los trabajos de campo de todas las investigaciones que se desarrollan en Catamarca. Si bien ese obstáculo fue superado, a continuación y como consecuencia de la pandemia de COVID-19, se vio imposibilitado, hasta el momento, el desplazamiento inter e intraprovincial en el territorio argentino. En consecuencia, se vieron interrumpidas las actividades planificadas para este período referentes a la continuidad de los planes de trabajo y la evaluación preliminar de los resultados.

\section{TAPSO: EL PASADO ES PRESENTE}

En la zona de Tapso las actividades desarrolladas han sido diversas. A fines de 2015 abre sus puertas el "Museo Histórico y Cultural de la Municipalidad de Tapso (figura 4) con el objetivo de preservar y dar a conocer el patrimonio cultural local. Para lograrlo, la municipalidad contó con el asesoramiento de diferentes profesionales ${ }^{2}$. Nuestro equipo estuvo a cargo del diseño y armado de la sala de arqueología, sustentado en las investigaciones propias en el área e implementando recursos audiovisuales (impresiones, gigantografías, videos, textos breves, entre otros) y didácticos (rompecabezas temáticos, esténciles con motivos rupestres, soportes para actividades expresivas). Incluimos también, entrevistas a referentes tradicionales de la comunidad y la exhibición de materiales aportados voluntariamente por vecinos.

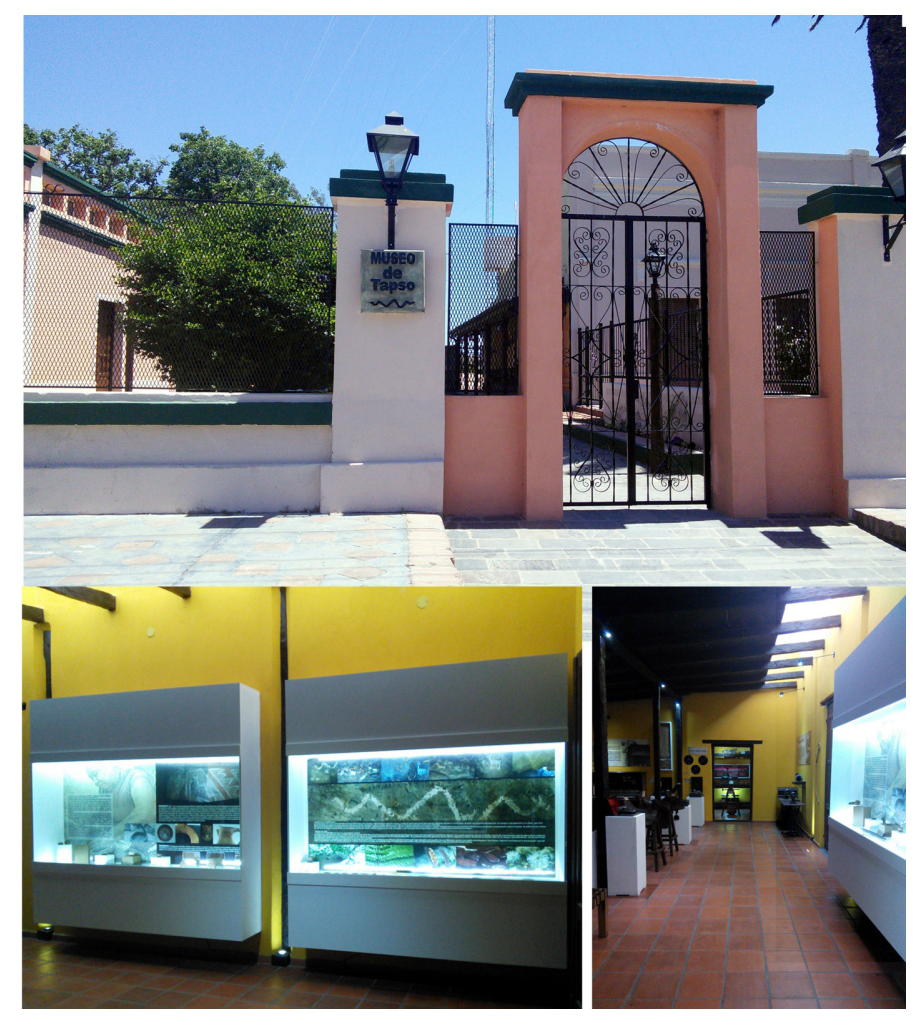

Figura 4: Vista del exterior del Museo de Tapso y de sus salas de exhibición.

$2 \quad$ El proyecto general "Casa de la Cultura y Museo de la ciudad de Tapso" fue elaborado por la Lic. Silvina Acevedo y la Arq. Alejandra Martínez. 
Asimismo, a partir de la propuesta de la Municipalidad de Tapso, realizamos la capacitación de guías con el fin de orientar las visitas y optimizar la información exhibida en el museo, así como la generación de fuentes de trabajo para los residentes y la formación de recursos humanos para la gestión y promoción patrimonial. El grupo de asistentes a la capacitación estuvo conformado por siete jóvenes tapseñas, cuyo nivel educativo formal alcanzaba el nivel secundario completo. Considerando que se trataba de su primer acercamiento a la temática arqueológica y patrimonial, en todo momento se buscó un intercambio fluido con las participantes, ajustando el contenido a sus inquietudes y sugerencias.

El programa de capacitación consistió en tres ejes temáticos. El primero de ellos se centró en arqueología general y del Noroeste Argentino (conceptos generales de la disciplina y específicos del área). En el segundo eje se abordaron los conceptos de patrimonio cultural y arqueológico, considerando la legislación que los rige en Argentina. Por último, se trabajaron las definiciones de museo, colección y guión museístico; luego se planteó una somera introducción a la estructura organizativa diseñada para el museo y a las formas de realización de las visitas guiadas y recorridos (ICOM 2007; Mairesse y Desvallées 2010). Aplicamos para ello diversos recursos didácticos, partiendo de las experiencias de los participantes con respecto al patrimonio arqueológico zonal (hallazgos, lugares, relatos y hechos conocidos por la comunidad).

En el año 2017 se inició un ciclo anual sobre arqueología, para la comunidad educativa de Tapso, con el objetivo de comunicar nuestras investigaciones $y$, al mismo tiempo, enriquecernos con las miradas locales. Este ciclo Ilamado "Taller de Arqueología"3 (figura 5), se centró en dos ejes: “¿Qué es la arqueología?" y "Tapso: pasado y presente". Allí desarrollamos presentaciones audiovisuales e interactivas, actividades expresivas (dibujos y relatos) y lúdicas (rompecabezas, esténciles, materiales gráficos, etc.). A partir de estas propuestas pedagógicas trabajamos la noción de arqueología, así como sus métodos, resultados y vinculaciones con la sociedad. El otro eje, se trató sobre la (pre) historia de Tapso, su nexo con el presente y sus proyecciones en un horizonte futuro.

En el 2018 continuamos con el Taller, incorporando estudiantes de otros niveles, para lo que se implementaron distintas estrategias didácticas. En este caso la actividad se centró en el procesamiento inicial de materialidades arqueológicas (preparadas para la ocasión) y su interpretación. Así, por ejemplo, luego de la presentación y motivación inicial, las niñas y niños de primaria desarrollaron una actividad lúdica en grupos que simuló el trabajo de campo y de laboratorio del arqueólogo. En tanto, los estudiantes secundarios trabajaron con una propuesta similar, pero sin materialidades; más bien, se basó en la transmisión audio-visual y dialógica. De esta manera, participaron de una exposición que sirvió como disparador para un intercambio activo entre alumnos/as, docentes e investigadores, debatiendo interpretaciones e interrogantes sobre la arqueología y el patrimonio cultural local.

3 Tanto el Taller como la Capacitación de guías estuvieron a cargo de docentes de distintos niveles que integran nuestro equipo de trabajo. 

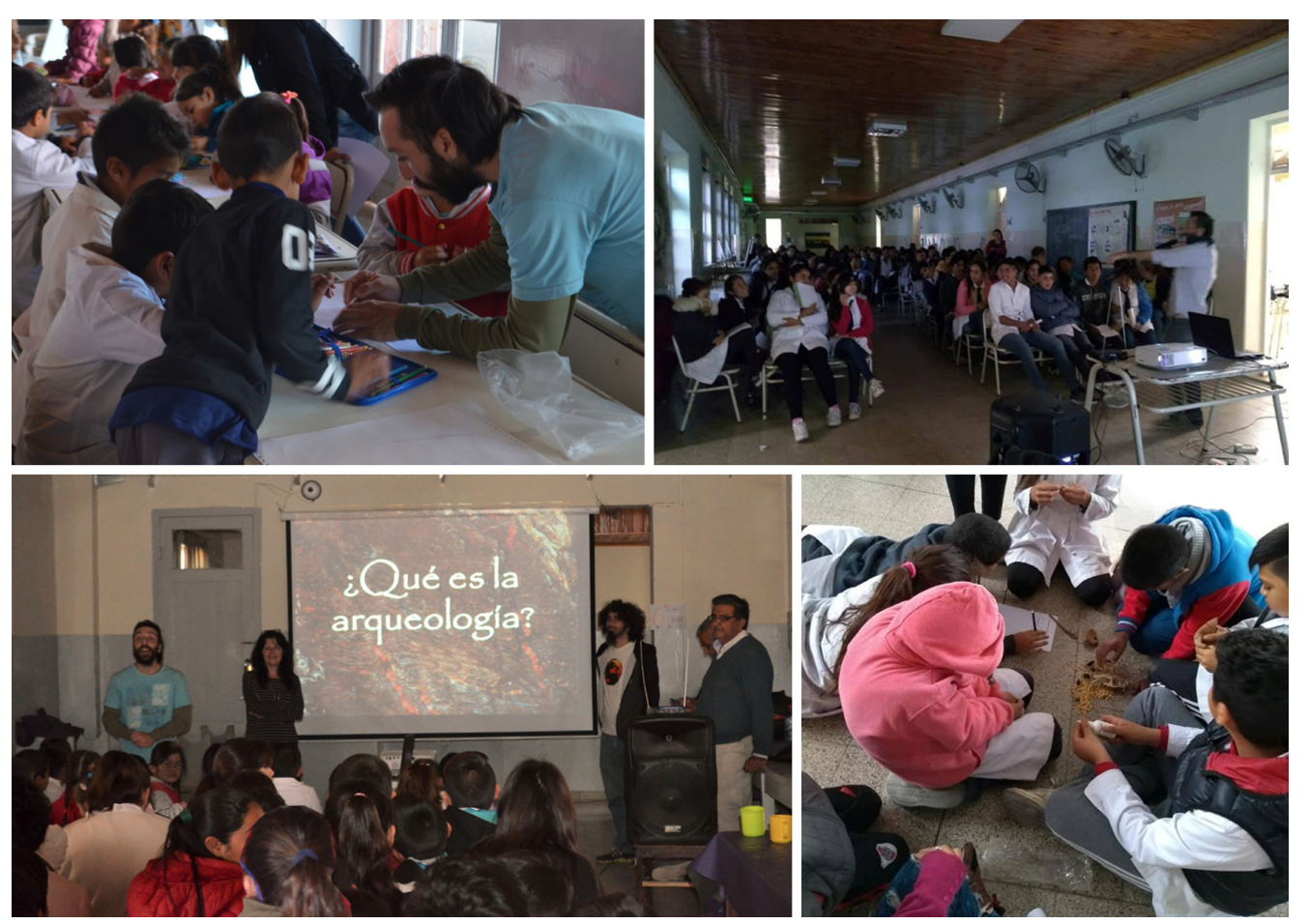

Figura 5: Actividades sobre el patrimonio cultural con la comunidad escolar de Tapso.

Los diversos materiales elaborados para estas actividades, así como libros de divulgación, pasaron luego a formar parte de los recursos del Museo para su potencial uso en las visitas escolares. Los posters (figuras 6 y 7) no solo vinculan gráficamente el presente y pasado local, sino que también incorporan los dibujos realizados por el alumnado de los talleres y hoy forman parte del acervo didáctico de la escuela.

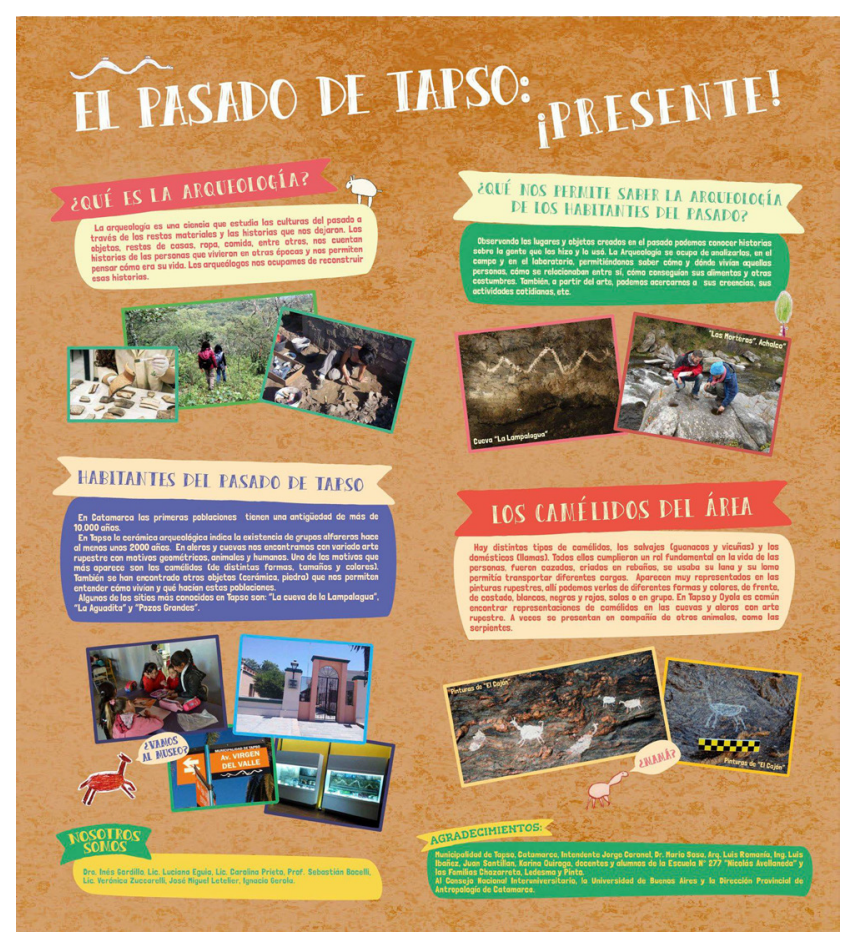

Figura 6: Póster elaborado para la escuela de Tapso.

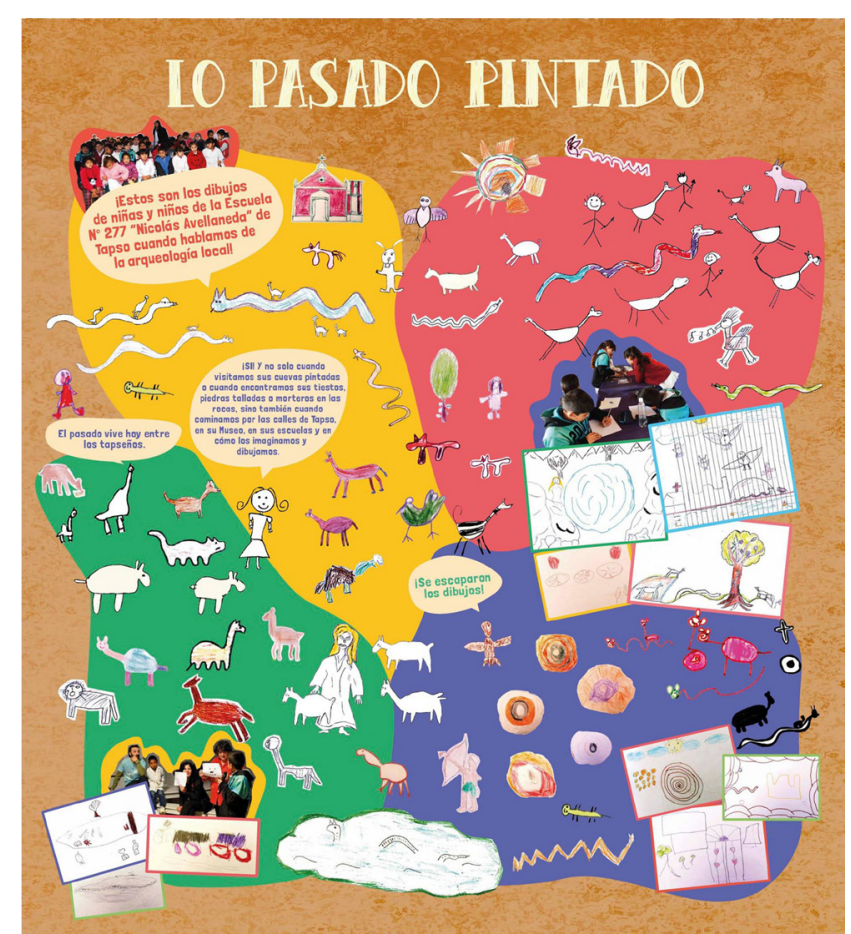

Figura 7: Póster basado en los dibujos de las niñas y niños realizados durante las actividades en la escuela de Tapso. 
En paralelo, diferentes actores de la comunidad de Tapso participaron activamente del trabajo arqueológico (figura 8). En muchas ocasiones nos acompañaron en los recorridos y registros. Por ejemplo, Luciano Pinto, un joven habitante en la zona rural de Pozos Grandes, en varias temporadas nos guió en la prospección con su conocimiento del paraje y de su aguda observación de sitios arqueológicos. Él, como otros lugareños, colaboró también con las tareas de relevamiento y con sus ideas, interpretaciones y relatos sobre la gráfica rupestre. En julio del 2017 organizamos un grupo de exploración para localizar más sitios en los campos de La Aguadita. El mismo estaba compuesto por un trabajador rural, una guía del Museo, funcionarios municipales e integrantes de nuestro equipo. Juntos relevamos nuevos sitios de arte rupestre y restos de construcciones precolombinas e históricas. Asimismo, compartimos jornadas en las casas de distintos pobladores, quienes nos han enseñado pequeñas colecciones halladas en sus campos, así como muchas vivencias y relatos del lugar.
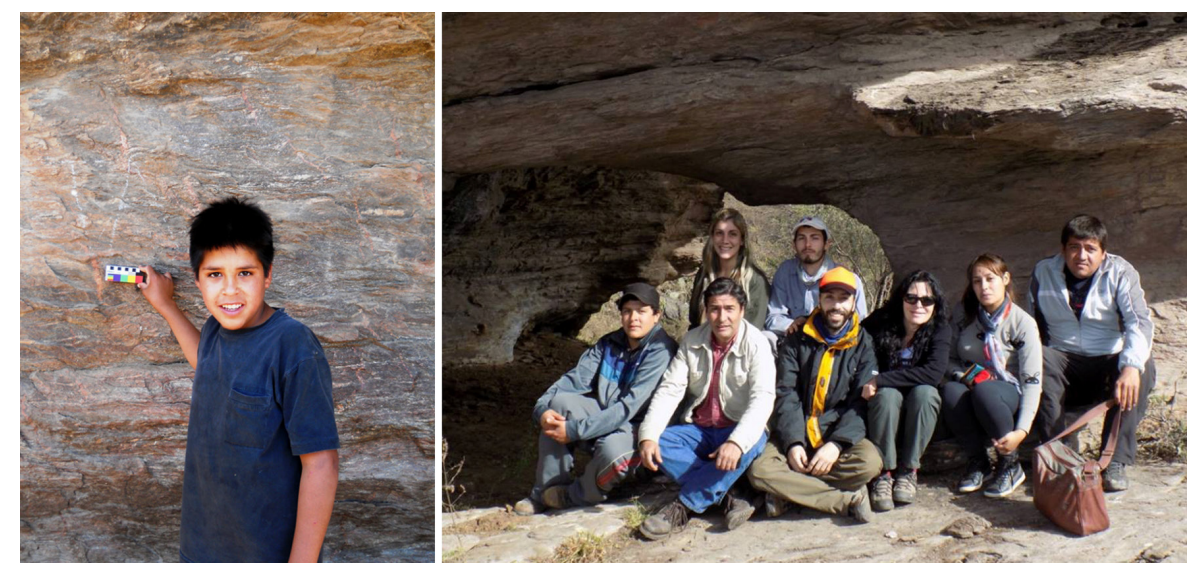

Figura 8: Luciano Pinto participando de tareas arqueológicas en Pozos Grandes (izquierda) y grupo de trabajo integrado por arqueólogos y pobladores en La Aguadita 3 (derecha).

Por otra parte, por iniciativa de la municipalidad y comunidad de Tapso los carteles que llevan el nombre de las calles contienen la figura de la Lampalagua, una representación precolombina que domina el interior de una de las cuevas. La misma se repite en cada esquina, así como en un mural callejero de grandes dimensiones en el centro del poblado, donde aparece junto a otras reproducciones del arte rupestre local (Figura 9). De esta manera, tales íconos adquieren valor en el paisaje tapseño actual como elementos identitarios de la comunidad.

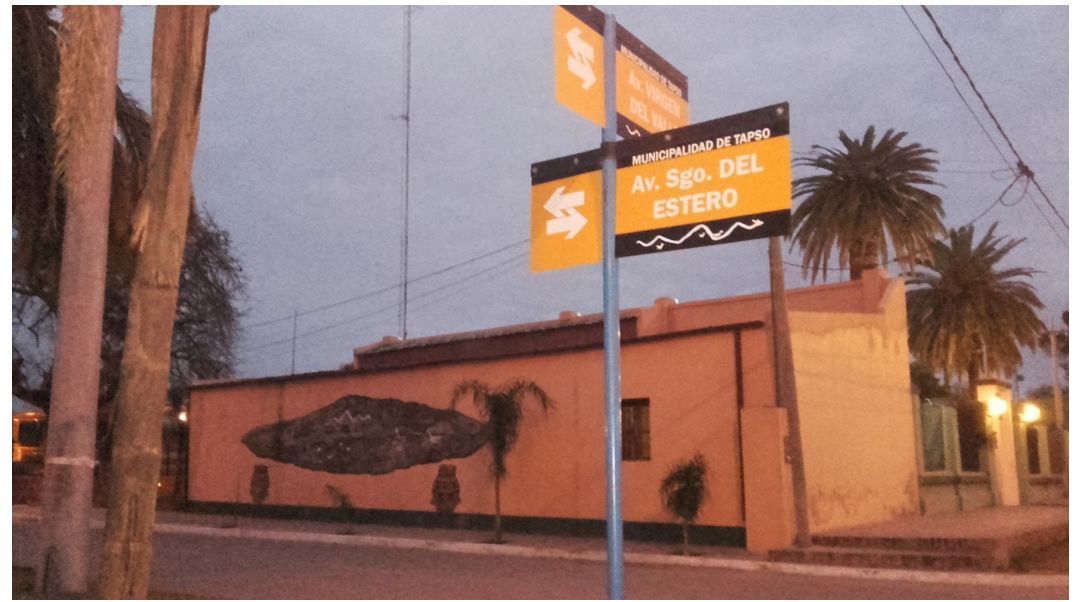

Figura 9: Esquina de Tapso con carteles indicadores de las calles y mural, con la reproducción de La Lampalagua. 


\section{NUESTRA EXPERIENCIA EN GUAYAMBA}

El proyecto de activación y puesta en valor del patrimonio funciona aquíde manera independiente al de Tapso, y se encuentra en diferente grado de avance. EI ASETUR -ver supra-sufrió importantes dilaciones en la ejecución debido a cuestiones administrativas ajenas a nosotros. Este proyecto es una presentación conjunta con el equipo de investigación arqueológica ${ }^{4}$ dirigido por Marcos Quesada, con el cual compartimos áreas de trabajo. También se vieron involucrados otros actores, organismos de turismo nacionales y provinciales, autoridades locales y profesionales independientes. Aun cuando el trabajo conjunto implica el esfuerzo adicional de generar acuerdos, la labor mancomunada posee grandes ventajas. Entre ellas podemos destacar las contribuciones conjuntas a la investigación de base, la generación de una mirada más amplia e interdisciplinaria sobre el patrimonio y el interés por la preservación y puesta en valor del mismo.

Uno de los frutos de este trabajo fue el diseño y montaje de la muestra "El Alto antes de El Alto" (figura 10), inaugurada en febrero de 2020. Consiste en un centro de interpretación, montado en la localidad de Guayamba, donde se narran los diversos modos de realización de las manifestaciones rupestres de la localidad cercana de Oyola. Se profundiza en las experiencias de sus hacedores y visitantes, incorporando reflexiones sobre la vida cotidiana, las viviendas, lugares de cultivo y relación con los vecinos. La muestra contiene también un panel orientado a reforzar las condiciones para proteger el patrimonio y un sector de participación interactiva y lúdica, dedicado a las infancias.

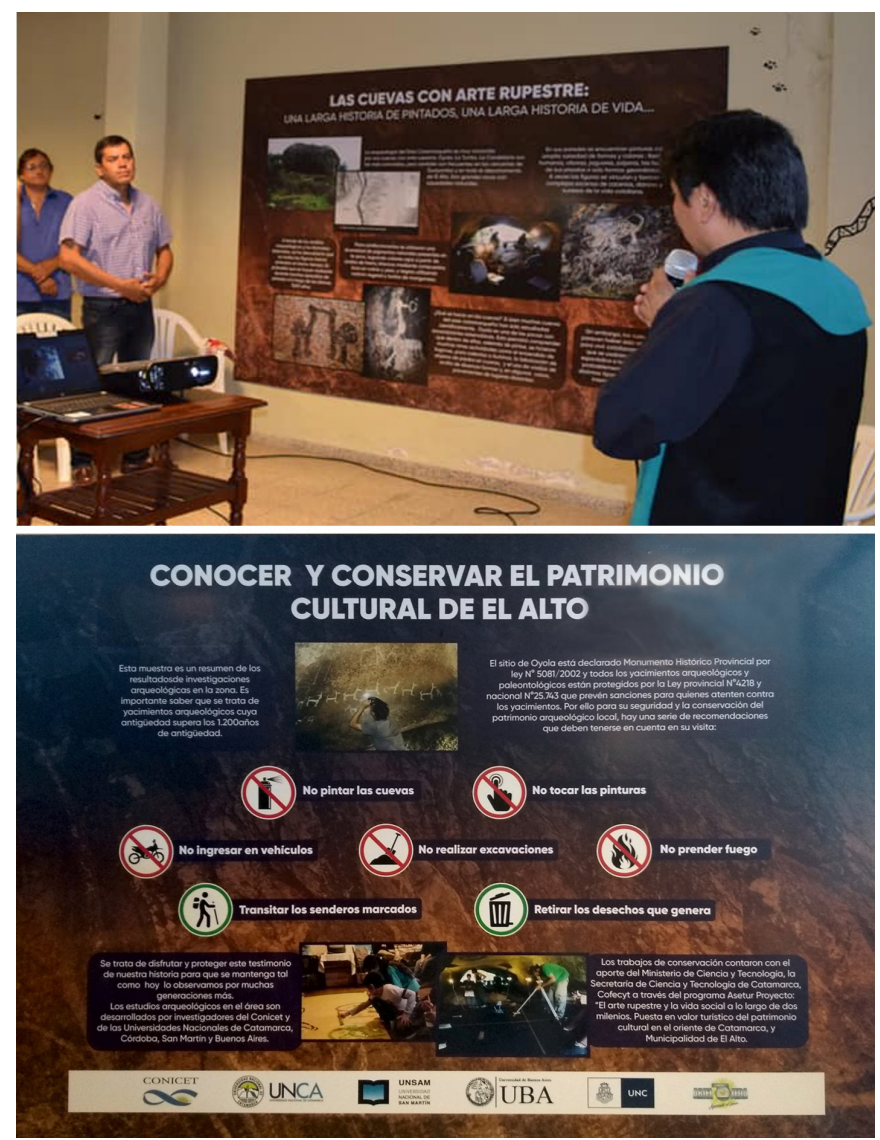

Figura 10: Inauguración de la muestra "El Alto antes de El Alto" (arriba, foto de la Municipalidad) y uno de sus murales sobre preservación del patrimonio (abajo).

Equipo Interdisciplinario El Alto-Ancasti. 
Nuestra idea es que esta exhibición pueda tener un carácter dinámico que permita incorporaciones y reformulaciones. Por ejemplo, actualmente, se aborda principalmente la temática de Oyola -localidad muy conocida y promocionada-, y la intención es que este eje pueda, luego, virar dando más espacio a lugares y experiencias propias de Guayamba (figura 11), revalorizando lo inmediato que no se encuentra aún incorporado con el mismo peso.
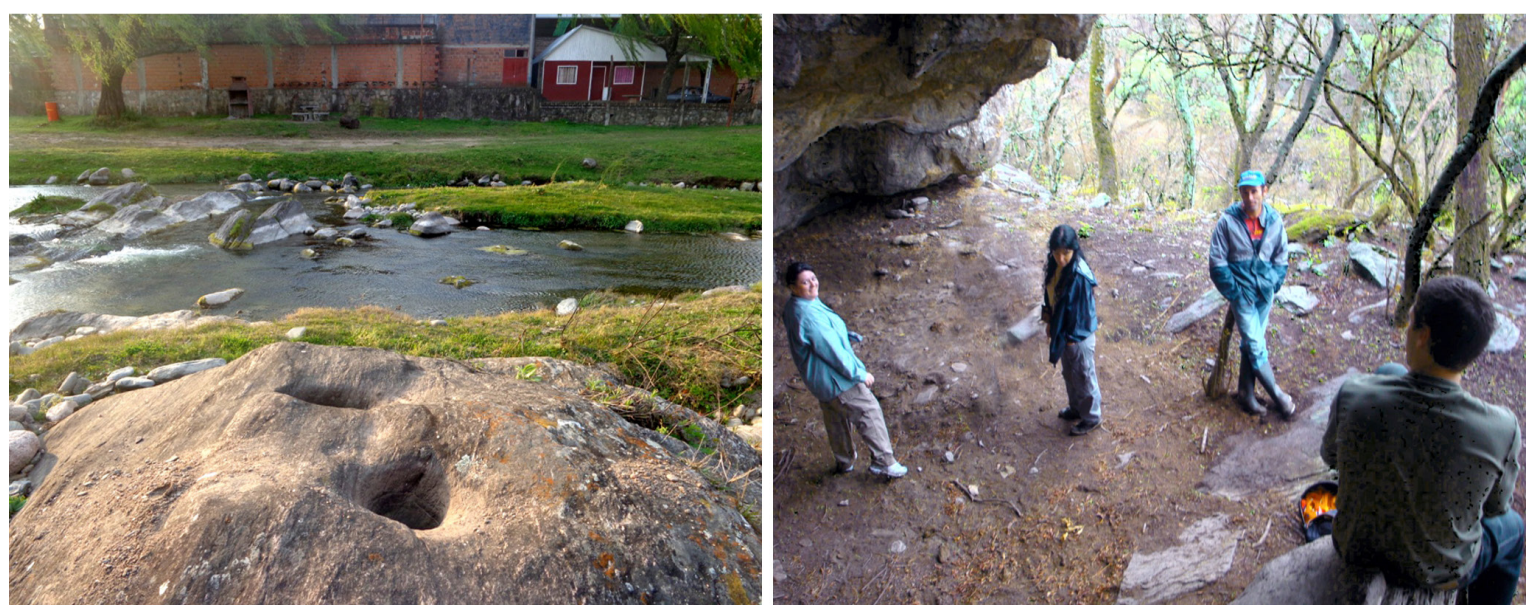

Figura 11: Morteros junto al río en la localidad de Guayamba (izquierda) y sitio Casa Pintada con integrantes del equipo junto con uno de los dueños de campo (derecha).

En el transcurso de los años nuestra relación con la comunidad se ha profundizado, los vínculos con los vecinos se hicieron más estrechos, fortaleciendo el diálogo e intercambiando experiencias, ampliando nuestra visión del patrimonio. Algunos nos compartieron sus saberes sobre la fauna y la flora, y sus relatos acerca de lugares y hechos memorables. También quisieron conocer nuestra opinión acerca de objetos arqueológicos que resguardan en sus casas ${ }^{5}$, o bien mostraron preocupación por la protección de materiales arqueológicos hallados en la ejecución de obras públicas o privadas.

Esto refuerza la idea de que los habitantes están interesados en conservar y preservar el patrimonio, y se encuentran abiertos a la posibilidad de realizar rescates. También, fuimos contactados con diversas consultas por la inicial Red de Turismo que se está formando entre comerciantes y emprendedores de la zona. Asimismo, con el tiempo hemos logrado una relación más fluida con las autoridades, encontrando mayor recepción a nuestras iniciativas. Por ejemplo, para el año 2020 la localidad iba a ser la receptora del $4^{\circ}$ TASA, ofreciendo ventajas de alojamiento e instalaciones públicas.

En el futuro, nos proponemos continuar y profundizar estas tareas que debieron posponerse. Como en Tapso, también aquí aspiramos a realizar talleres en las escuelas y otros espacios de la comunidad, siempre en el marco de una construcción compartida del conocimiento y vinculando las narrativas sobre el pasado propias y de los habitantes locales.

$5 \quad$ Nuestra propuesta es que tales materiales formen parte del centro de interpretación, propiciando el protagonismo de la comunidad en la recreación de ese espacio. 


\section{CONCLUSIONES}

A lo largo de este trabajo hemos hecho un breve raconto de las trayectorias de dos proyectos de activación patrimonial desarrollados en localidades cercanas pero diferentes. Estos recorridos nos despertaron renovadas inquietudes sobre la relación entre el quehacer arqueológico, el patrimonio, y las comunidades. Para realizar este trayecto planteamos tres ejes principales: una noción ampliada de patrimonio arraigada en el paisaje cultural como articulador; una construcción co-participativa con la comunidad local y diferentes actores institucionales; y finalmente, una preocupación sobre cómo integrar estos dos ítems con el turismo como práctica, y las dificultades que impone una complejización del modelo de patrimonialización.

Entender el patrimonio en términos de paisaje, nos invita a ampliar nuestra mirada, desplazándola de los sitios tradicionalmente puestos en valor hacia otros componentes del paisaje, otros "hitos". Esto también conlleva otras preguntas ¿cómo era la vida cotidiana de los habitantes de este sector? ¿cómo eran sus viviendas? ¿cómo producían sus alimentos? ¿qué continuidades y rupturas plantea la historia del paisaje en la Sierra El Alto-Ancasti? La perspectiva de trabajo adoptada ha permitido poner en escena diversas historias, interpretaciones y valoraciones de lo local, de quienes habitan y transitan estos espacios cotidianamente. En este sentido, hemos podido acercarnos al modo en el que los paisajes del pasado se incorporan en las sociedades siguientes, integrando, reinterpretando y superponiendo lo prehistórico y lo contemporáneo (Ballesteros et al. 2005).

En este sentido entendemos que el patrimonio lo constituyen los restos de la memoria, un foro de esta, su representación. Se construye desde el horizonte de la actualidad y la identidad, supeditada a la política del presente. Entonces, la memoria no recuerda todo, ni todo por igual. Debemos preguntarnos entonces por aquello que desconoce, ignora y otras veces oculta. ¿Qué pasa con el olvido? El patrimonio cultural es la huella de la memoria y del olvido, del presente y del pasado. Está constituido por las ruinas de la memoria, lo que recordamos y lo que decidimos olvidar de nosotros mismos (Criado 2012). También es importante reflexionar acerca de ¿qué es lo museable? En las salas de exposición y en el paisaje, ¿qué y cómo seleccionamos los materiales, imágenes y sitios a exhibir?

La relación pasado-presente en nuestra área específica está dada por el lugar. Los miembros de la comunidad no se identificaban, en principio, con el patrimonio arqueológico y no lo percibían como elemento identitario debido a su carácter criollo y a la discontinuidad histórica entre este presente y el pasado prehispánico (Pizarro 2006). Sin embargo, existe una lógica que asume su presencia o preexistencia como parte del paisaje propio y que a partir de eso y de la atribución de valores históricos, estéticos, simbólicos, etc., se lo puede reconocer como una herencia (Gordillo 2017). Es entonces cuando se los puede poner en valor y abrir al uso público.

El vínculo entre el trabajo arqueológico y las comunidades posibilitó una mayor multivocalidad y permitió enriquecer nuestras interpretaciones con otros saberes y modos de ver el mundo. Por ejemplo, en Tapso tanto los trabajos de campo, como el TASA, iniciaron un camino de incorporación a la vida pública de la arqueología que refleja una acción simbiótica entre nuestro equipo y los 
habitantes. Esta se materializa en el museo, en los carteles de la calle y en el mural, replicando e incorporando a la cotidianidad los motivos rupestres. A su vez, las actividades de difusión y comunicación han permitido continuar con una dinámica recursiva, problematizando el patrimonio. Esto se manifiesta en las producciones del alumnado y en los posters generados a partir de ellas.

En cuanto a la muestra de Guayamba, nuestra expectativa es que funcione como un disparador, un punto de partida para seguir compartiendo experiencias e incorporar narrativas sobre los lugares cotidianamente transitados, las prácticas tradicionales y habitualmente realizadas, los objetos y sus biografías. Tal es el caso de los morteros, que se encuentran atravesando todos los caminos, en la calle principal, en las márgenes del río donde la gente se baña, en las casas.

La noción de paisajes culturales requiere trabajar en la protección y difusión del patrimonio a una escala más amplia, desde estrategias que contemplen su complejidad y posibilidades (Ballesteros et al. 2005). No solo apuntamos a una construcción compartida del conocimiento, sino también a una gestión y conservación conjunta. Desde allí, es necesario analizar la vulnerabilidad de los paisajes culturales y trazar, en conjunto, estrategias y políticas de actuación en ellos, evaluando previamente sus consecuencias. En este escenario, los estudios de impacto, siempre desde esta perspectiva holística y dialógica, se constituyen como otro de los principales aportes de la arqueología a la gestión patrimonial.

Esto nos deja lugar al último, pero no menos importante punto: el vínculo entre patrimonio y turismo. En principio, consideramos esencial que las comunidades puedan gestionar su patrimonio en pos de un desarrollo sustentable y beneficioso. La situación frente al turismo es diferente en las dos localidades, por un lado, Guayamba es un foco turístico estival posicionado y, por el otro, Tapso, es una localidad que está orientando acciones para posicionarse como un destino posible y atractivo, sin embargo, ambas localidades quieren potenciar su oferta turística mediante la puesta en valor del patrimonio arqueológico e histórico. Creemos que para desarrollar un producto turístico sostenible debemos retomar y reforzar algunos de los puntos antes abordados, una construcción compartida de las narrativas sobre el pasado, la gestión orientada a la protección y valoración del patrimonio como recurso no renovable, rescatar la tradición oral, historia escrita y cultura material, que refuercen la identidad, valores y costumbres locales. Este proyecto respalda un modelo turístico sostenible y autogestionado por la comunidad, que involucre a sus miembros y que fomente la integración y participación.

\section{AGRADECIMIENTOS}

Al Sr. Intendente Ariel Ojeda, secretaria Mariana Rodriguez y equipo; al Delegado Nardelli; Ing. Seven y flia, y a los vecinos Zenón, familia Bulacio, Kelo, Ana M. Fernández, Chani Gomez. En Tapso al Sr. Intendente Sosa y equipo; ex intendente Coronel y equipo, Arq. Romanía, Ing. Ibañez, al Sr. Santillán y personal de la hostería, familias Ledesma, Chazarreta y Pintos; a María, su esposo y Carina; a Don Pili; a las autoridades de la Escuela "Nicolás Avellaneda", Ana Silva y Patricia Pereyra y a sus docentes y alumnos. Y por supuesto a todos los vecinos del Depto. El Alto que brindaron su apoyo y amabilidad. Este trabajo fue realizado con el apoyo del CIN y el MINCYT. 


\section{BIBLIOGRAFÍA}

ALANIZ, Hugo. Historias y Vivencias: Tapso, Catamarca. San Fernando del Valle: Editorial Mann, 2010.

ALONSO GONZÁLEZ, Pablo. Patrimonio y ontologías múltiples: hacia la coproducción del patrimonio cultural. En: GIANOTTI, C.; BARREIRO, D. y VIENNI, B. (eds.). Patrimonio y Multivocalidad. Teoría, práctica y experiencias en torno a la construcción del conocimiento en Patrimonio. Montevideo: Ediciones Universitarias, 2016, p. 179-198

ARANTES, Antonio. Diversity, Heritage and Cultural Politics. Theory, Culture \& Society, 24, p. 290296, 2007.

ARgÜELlO GARCíA, Pedro M. Presentación. En: Gnecco, C. El señuelo patrimonial. Pensamientos post-arqueológicos en el camino de los incas. Tunja: Maestría en patrimonio cultural, 2019, p. 7-11

ASCHERO, Carlos, Victor ATALIVA, Lorena COHEN, Sara LÓPEZ CAMPENY, Carolina SOMONTE. Arqueología e identidad o identidad de la arqueología en la comunidad indígena de Amaicha del Valle (Tucumán, Argentina). Textos Antropológicos, La Paz: Universidad Mayor de San Andrés vol.15, número 2, p. 263-276, 2005.

BALLESTEROS, Paula, OTERO VILARIÑO, Carlos y VARELA-POUSA, Rocío. Los Paisajes Culturales desde la arqueología: propuestas para su evaluación, caracterización y puesta en valor. ArqueoWeb 7(2), 2005 https://webs.ucm.es/info/arqueoweb/numero-7-2.html\#7-2

BARRETT, James. Agency, the Duality of Structure, and the Problem of the Archaeological Record. En: HODDER, I. (ed.). Archaeological Theory Today. Cambridge: Polity Press. 2001, p. 141-164.

BELLELLI, Cristina. Arqueología y patrimonio. Reflexiones desde la práctica. Comechingonia, Córdoba: 16, p. 273-279, 2012.

BOCELLI, Sebastián. Motivos para dibujar la roca: un primer acercamiento al arte rupestre de La Aguadita (Tapso, Catamarca). Comechingonia, Córdoba: 20: 2 p.105-126, 2017.

CRIADO BOADO, Felipe. Arqueológicas: la razón perdida. La construcción de la inteligencia arqueológica. Barcelona: Bellaterra, 2012.

DELFINO, Daniel, Andres BARALE, Alejandro DÍAZ, Sabine DUPUY, Valeria ESPIRO, Gustavo PISANI. El Museo Integral de la Reserva de Biosfera de Laguna Blanca como soporte y vehículo de confrontaciones discursivas, de prácticas académicas y campesinas. Actas del IV Encuentro de Museos Universitarios del Mercosur. I Encuentro de Museos Universitarios Latinoamericanos y del Caribe. Santa Fe: Museo Histórico, Universidad Nacional del Litoral. p. 150-161. 2013

EGUIA, Luciana. La vida en la selva: una aproximación a la estructuración del espacio doméstico en el extremo oriental de Catamarca. (Doctorado), Facultad de Filosofía y Letras, UBA, Buenos Aires, 2019.

FUNARI Pedro, CARVALHO, Aline. Inclusión en la arqueología pública brasileña: apuntes sobre prácticas colaborativas. Multivocalidad y Activaciones Patrimoniales en Arqueología: Perspectivas desde Sudamérica. Fundación de Historia Natural Félix de Azara. 2014. pp. 193-216 
GONZÁLEZ-RUIBAL, Alfredo; GONZÁLEZ Pablo A. y CRIADO-BOADO Felipe. En contra del populismo reaccionario: hacia una nueva arqueología pública. Chungara, p. 1-7, 2018

GORDILLO, Inés. La noción de paisaje en arqueología. Casos de estudio y aportes al patrimonio. Jangwa Pana, Santa Marta: Facultad de Humanidades, Universidad del Madalena, vol. 13, p. 195-208, 2014.

GORDILLO, Inés. Arqueología, Patrimonio y Memoria. Propuestas y experiencias en el oriente de Catamarca (Argentina). En: Diversidad e identidad: Contextos del patrimonio cultural edificado.Mérida: Universidad Autónoma de Yucatán, p. 175-189, 2017.

GORDILLO, Inés y VAQUER, José M., La Espacialidad en Arqueología: Enfoques, Métodos y Aplicación. Quito: Abya-Yala, 2013.

GORDILLO, Inés; ZUCCARELLI, Verónica y Luciana EGUÍA. Las casas del sol naciente: Arqueología de la vertiente oriental de El Alto-Ancasti. En: VENTURA B., G. ORTIZ y M.CREMONTE (eds). Arqueología de la vertiente oriental Surandina: interacción macro-regional, materialidades, economía y ritualidad. Buenos Aires: SAA, p. 111-134, 2017.

GHECO, Lucas, El laberinto de las paredes pintadas. Una historia de los abrigos con arte rupestre de Oyola, Catamarca.(Doctorado) Facultad de Filosofía y Humanidades. Universidad Nacional de Córdoba, Córdoba, 2017.

GNECCO, Cristóbal. El señuelo patrimonial. Pensamientos post-arqueológicos en el camino de los incas. Tunja: Maestría en Patrimonio Cultural, 2019.

HERNÁNDEZ RAMÍREZ, Javier. El patrimonio activado. Patrimonialización y movimientos sociales en Andalucía y la ciudad de México. Dimensión Antropológica 14, p. 7-44, 2007.

ICOM. Cómo administrar un museo. ICOM-UNESCO. 2007

INGOLD, Tim. The Perception of the Environment. Essays on Livelihood, Dwelling and Skill. Londres: Routledge, 2000.

JOFRÉ Carina, Soledad BIASATTI, Gonzalo COMPAÑY, Gabriela GONZÁLEZ, Soledad GALIMBERTI, Nadine NAJLE, Pablo AROCA. La Cayana: entre lo arqueológico y lo cotidiano. Tensiones y resistencias en las versiones locales del "patrimonio arqueológico" en el norte de San Juan: Relaciones, Buenos Aires: Sociedad Argentina de Antropología XXXIII, p. 181-208. 2008.

KORSTANJE, M. Alejandra, Jorgelina GARCÍA AZCARATE y Patricia ARENAS. Intercultural processes of territory-heritage recovery and management in the Calchaquí valleys, Tucumán, Argentina. Nuevo Mundo Nuevos Mundos, Paris: École des hautes études en sciences sociales, 2013, p. 1-36.

LLAMAZARES, Ana. Arte rupestre en la cueva de La Candelaria, provincia de Catamarca. Publicaciones Arqueología, Córdoba: 50, p. 1-26, 1997/98.

LONDOÑO, Wilhem. Más allá del Patrimonio. En: RIVOLTA, M., M. MONTENEGRO, L. MENEZES FERREIRA, J. NASTRI (Eds). Multivocalidad y Activaciones Patrimoniales en Arqueología: Perspectivas desde Sudamérica. Buenos Aires: Fundación de Historia Natural Félix de Azara. 2014, p. 155-168.

MAIRESSE, François y André, DESVALLÉES. Conceptos claves de museología. Paris: Armand Colin, 2010. 
MC DAVID, Carol. Community Archaeology. En: SMITH, C. (ed.). Encyclopedia of Global Archaeology. Nueva York: Springer Science \& Business Media, 2014, p. 1591-1599.

NAZAR, Domingo Carlos. Parque Arqueológico La Tunita. Puesta en valor integral del arte rupestre de la Sierra de Ancasti, provincia de Catamarca, República Argentina. Tesis para optar por el grado de Magíster en Conservación del Patrimonio, Universidad Internacional de Andalucía. Catamarca, 2003

PIZARRO, Cynthia A. "Somos indios civilizados". La (in)visibilización de la identidad aborigen en Catamarca. Anuario de Estudios en Antropología Social, Buenos aires: IDES, p. 179-198, 2006.

PRATS, Llorenç. Antropología y Patrimonio. Barcelona: Ariel, 2004.

SHACKEL, Paul. Stakeholders and community participation. En SMITH, C. (ed.) Encyclopedia of Global Archaeology. Nueva York: Springer Science \& Business Media, 2014 p.6994-6998.

RECALDE, Andrea. Representaciones en contexto. Características del Paisaje rupestre de Cerro Colorado (Sierras del Norte, Córdoba, Argentina). Relaciones de la Sociedad Argentina de Antropología, Buenos Aires: XL (2):523-548, 2015.

SOLER SEGURA, Javier. Redefiniendo el registro material. Implicaciones recientes desde la Arqueología del Paisaje anglosajona. Trabajos de Prehistoria, 64 (1), p. 41-64, 2007.

THOMAS, Julian. Archaeologies of place and landscape. En: HODDER, I. (ed.).Archaeological Theory Today. Cambrige: Polity press, 2001, p. 165-186.

TRONCOSO, Alejandra. Patrimonio, turismo y lugar: selecciones, actores y lecturas en torno a la Quebrada de Humahuaca (Jujuy, Argentina) como Patrimonio de la Humanidad. Cuadernos de Turismo 25: 207-227, 2010.

VAQUER, Jose M. y Yamila CÁMERA. Las relaciones entre el paisaje, las narrativas y la praxis arqueológica en Cusi Cusi (Rinconada, Jujuy): una mirada hermenéutica. Revista del Museo de La Plata, La Plata: 3 (1): 38-56, 2018.

ZUCCARELLI, Veronica. Desde las cumbres a las yungas: múltiples escalas de las prácticas agrarias prehispánicas en la sierra de el Alto-Ancasti (Catamarca) durante el primer milenio A.D. (Doctorado), Facultad de Filosofía y Letras, UBA, 2020. 